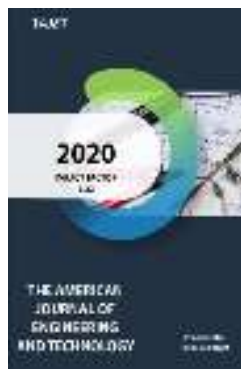

Journal Website: http://usajournalshub.c om/index,php/tajet

Copyright: Original content from this work may be used under the terms of the creative commons attributes 4.0 licence.

\section{Simulation Of The Turbulent Flow Effect On The Shore Spillways Of The Pachkamar Reservoirs}

\author{
Begimov Uktam Ibrogimovich \\ Assistant Of The Department “Algorithms And Mathematical Modeling” Of Tashkent \\ University Of Information Technologies Named After Muhammad Al-Khorezmi, Republic Of \\ Uzbekistan, Tashkent
}

Khudaykulov Savet Ishankulovich

Doctor Of Technical Sciences, Leading Researcher Of Scientific-Research Institute of Irrigation And Water Problems Of The Ministry Of Water Economy Of The Republic Of Uzbekistan, Tashkent

Usmonov Alisher Khabibulla Ugli

Assistant Of The Department “Algorithms And Mathematical Modeling” Of Tashkent University Of Information Technologies Named After Muhammad Al - Khorezmi, Republic Of Uzbekistan, Tashkent

\title{
ABSTRACT
}

A mathematical model of structures controlling the turbulent flows is considered. The shore spillways are investigated by constructing a free surface. Calculations of transition sections, turns, ramps-turns, scattering ramps in inclined drop structures with water flow rate from several cubic meters per second to several thousand, with a gradient from several degrees to $45^{\circ}$ or more, with a constant and variable gradient and width, with straight and curved axes are given.

\section{KEYWORDS}

Pachkamar reservoir, radial flow, transition sections, narrow flows, vertical angle of flow.

\section{INTRODUCTION}

The most common type of shore spillway is an inclined drop structure, the parameters of which change in a significant range in a short time depending on local conditions. In the Pachkamar reservoir, the shore spillway has an inclined drop structure with water flow rate from several cubic meters per second to several thousand, with a gradient from several degrees to $45^{\circ}$ or more, with a constant and variable gradient and width, with straight and curved axes.
The method of turbulent flows calculation within the transition sections, turns of the spillway end sections was obtained under the assumption that the vertical components of acceleration are small, therefore, the flow pressure is dissipated according to the hydrostatic law. In addition, the flows were considered in a channel with a flat bed or a bed of slight curvature. 


\section{MATERIAL AND RESEARCH METHODS}

The energy dissipation behind the inclined drop structure is carried out in a water cushion or a water jet throw from the ramp or console (Fig. 1).

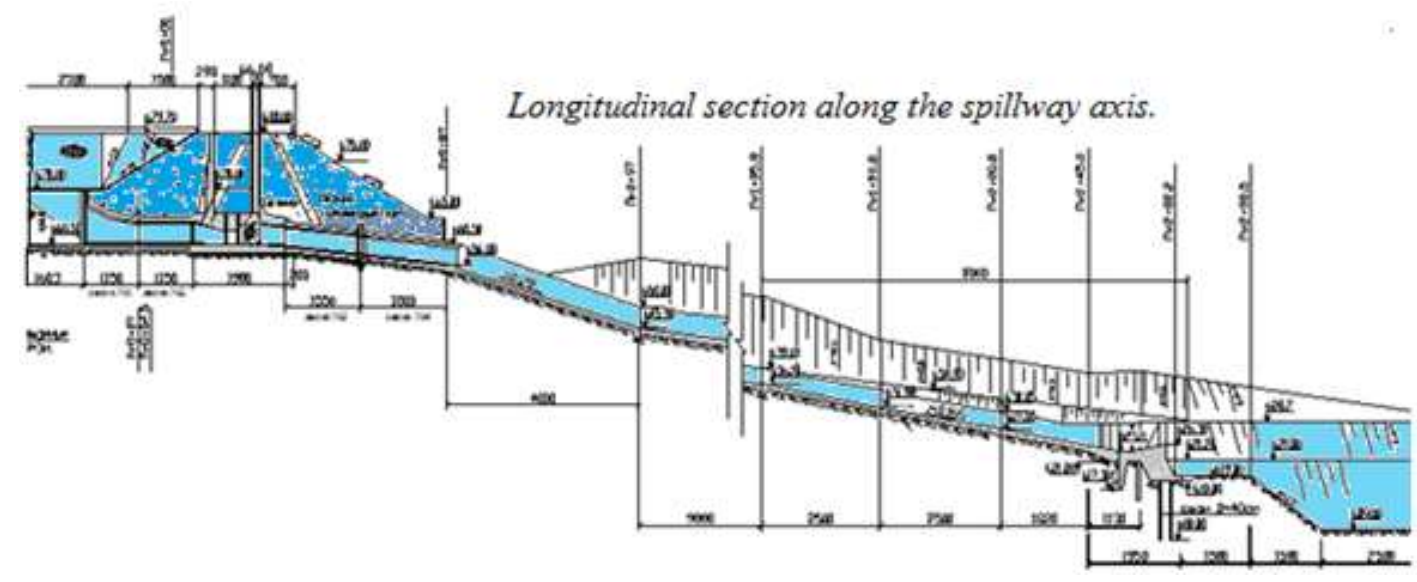

Fig. 1. Shore spillways of the Pachkamar reservoir.

In most cases (in the Tupalang, Pachkamar reservoirs) to control turbulent flows, it is necessary to build the structures that substantially deform the flow. The dissipating ramps, turns, in some cases transition areas, in which the bed has to be taken as a double curved one to avoid wave generation and to achieve a given dissipation of specific flow rates. Inside such constructions, the flow has a spatially curved free surface, and the flow pressure is not dissipated according to the hydrostatic law. Therefore, for the hydraulic calculation of structures of double curvature it is impossible to use either the methods of onedimensional hydraulics or the method of small height wave superposition. Such constructions can be calculated using the free surface equations and the continuity equations.

When designing structures of double curvature, the hydraulic calculation based on solving inverse problems of controlling turbulent flows is convenient to carry out with known planned flow shaping to be implemented. The tasks of this kind are often encountered in practice. In these cases, the double curvature is calculated in the following order.

1. The desired boundary of the turbulent flow in the plan is set $y_{\kappa}=y_{\kappa}\left(s_{\kappa}\right)$. This expression is normalized by introducing a characteristic length $L_{0}$ taken as $\left(s_{\kappa}\right)_{\text {макс }}$.

2. The required plan of surface lines (the flow plan) is accepted (set) in parametric form $y=y\left(s_{\kappa}, n\right)$.

Usually $L_{0}$ is set from the condition that $S_{\kappa}$ changes in the range $0-1$, that is, $L_{0}$ is taken equal to the length of the planned outer flow line within the integration domain, then the free surface equation has the form [1-3]: 


$$
\frac{d z}{d n}=A\left[B \frac{\partial z}{\partial s_{\kappa}}-\frac{C}{\frac{\partial^{2} z}{\partial s_{\kappa}^{2}}+D \frac{\partial z}{\partial s_{\kappa}}}+\frac{1}{\Pi}\right](1)
$$

Where

$$
\begin{array}{r}
A=\frac{d y}{d n}\left(\frac{d s_{\kappa}}{d s}\right)^{2}, B=\frac{d y}{d s_{\kappa}}, \\
C=\frac{d^{2} y}{d s_{\kappa}^{2}}-\frac{d y}{d s_{\kappa}} \frac{d s_{\kappa}}{d s} \frac{d^{2} s}{d s_{\kappa}^{2}}, \\
D=-\frac{d s_{\kappa}}{d s} \frac{d^{2} s}{d s_{\kappa}^{2}}, F=\left(\frac{d s}{d s_{\kappa}}\right)^{2}
\end{array}
$$

variable coefficients $A, B, C, D, F$ are specified after setting the plan of the boundary (outer) flow line $y_{\kappa}=y_{\kappa}\left(s_{\kappa}\right)$ and entering the parameter $n$.

3. The expressions of variable coefficients $A, B, C, D, F$ in equation (1) are specified.

4. The longitudinal profile of any of the surface flow lines is given by a suitable analytical expression. In this case, the requirements of a topographic nature, the need to spill the flow at a certain angle to the horizon, the need to couple two sections of the inclined drop structure having different slopes, etc. are taken into consideration. Usually, a longitudinal profile of the axial or boundary (outer) flow line is specified. In the latter case, $z_{\kappa}=z_{\kappa}\left(s_{\kappa}\right)$ is set.

5. The equation of the free surface is solved in coordinates $s_{k}=z n$ and the shape of the free surface corresponding to a given flow plan is determined.
6. The marks of the structure bed are determined using the continuity equation.

7. A transition from coordinates $s_{k}, n$ to $x$ and $y$ coordinates is done; it gives the shape of the free surface $z=z(x, y)$ and of the bed $z_{\text {bed }}=z_{\text {bed }}(x, y)$ in the form convenient for construction. Structures with a bed of double curvature should be used in cases where it is necessary to carry out a sharp shaping of a turbulent flow without significant wave generation on the free surface.

As already was indicated, the free surface equation (1) is a partial nonlinear differential equation of the second order with variable coefficients and of parabolic type. The domain of finding a solution to equation (1) in coordinates $s_{k}, n$ is usually a rectangle $0 \leq s_{k} \leq 1 \quad 0 \leq n \leq 1$ (Fig. 2).

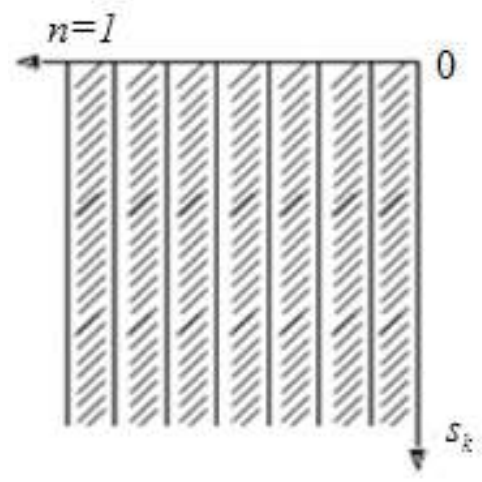

Fig.2. Equation (1) integration domain.

The boundary conditions of the free surface equation also include the slope, level and rate in the initial section of the structure $\left(s_{k}=0\right.$ ), in the general case, the variables along the section, i.e., depending on $n$, are: 


$$
\begin{gathered}
\left.\frac{\partial z}{\partial s_{k}}\right|_{s_{k}=0}=\left.i_{0}(n) \quad z\right|_{s_{k}=0}=z_{0}(n) \\
\left.\vartheta\right|_{s_{k}=0}=\vartheta_{0}(n)
\end{gathered}
$$

An expression specifying the longitudinal profile of any one of the surface flow lines (usually the boundary ones) is $z_{\kappa}=z_{\kappa}\left(s_{\kappa}\right)$. After setting the desired plan of surface lines in a parametric form $y=y\left(s_{\kappa}, n\right)$, the coefficients of the free surface $A, B, C, D, F$ are concretized and become some known functions of $s_{k}$ and $n$. In the general case, equation (1) cannot be implemented analytically due to the impossibility of mathematical solution. Therefore, it is solved, as a rule, numerically using computer technology. For this, it is necessary first to proceed to the equivalent equation of a free surface in an integro-differential form:

$$
\left.z=z_{k}+\int_{1}^{n} A\left[B \frac{\partial z}{\partial s_{k}}-\frac{C}{\frac{\partial^{2} z}{\partial s_{k}^{2}}+D} \frac{F+\left(\frac{\partial z}{\partial s_{k}}\right)^{2}}{F}\right] \frac{1}{\Pi}\right]
$$

Numerical solution is implemented according to the developed computational scheme $[1,3]$. A universal program in codes as applied to the use of computers is given in [2, 6]. In the particular case, if the planned flow shaping is relatively small, an approximate solution of equation (1) can be used, provided that the parameter $n$ is introduced in the

form $n=\frac{\alpha}{\alpha_{\kappa}}$ : 


$$
z\left\langle>z_{k}+y_{k}\left[\frac{d z_{k}}{d s_{k}} \frac{\cos \alpha_{k}}{\alpha_{k}} \ln \frac{\cos \alpha_{k}}{\cos \left(n \alpha_{k}\right)}+\frac{0,5 c\left(1-n^{2}\right)}{\frac{d^{2} z_{k}}{d s_{k}^{2}}} \frac{1}{1+\left(\frac{d z_{k}}{d s_{k}}\right)^{2}}+\frac{1}{\Pi}\right]\right.
$$

When determining the shape of the free surface, it is necessary to verify compliance with the following general provisions:
a) the flow must remain turbulent everywhere;
b) the longitudinal profile of the flow lines should not have sections that are convex flow becomes uncontrollable; upward and have a curvature equal to or greater than the curvature of the free fall trajectory, since in the opposite case the
c) for aerated flows, the calculation is carried out in the same way, however, the free surface is conventionally understood as the boundary between the water-air and water-drop layers.

The coordinates entering the equations are reduced to a dimensionless form by dividing them by a certain characteristic length ${ }^{L_{0}}$, for which we take the full length of the outer flow line $S_{k}($ at $n=1)$.

The transition sections of the inclined drop structures with a curved bed, where the

$$
y_{\kappa}=y_{k 0}+s_{k} \sin \alpha_{k o}
$$

The coordinate of any flow line is determined by the formula: []

$$
y=y_{\kappa} \frac{\operatorname{tg}\left(n \alpha_{k 0}\right)}{\operatorname{tg} \alpha_{k 0}}
$$


Denoting $\lambda=\frac{B}{b_{0}}$, for a symmetric plan of flow (the width of initial section is $\frac{b_{0}}{2}$ ) we obtain

$$
\lambda=\frac{\frac{b_{0}}{2}+L_{0} \sin \alpha_{k 0}}{\frac{b_{0}}{2}}
$$

hence

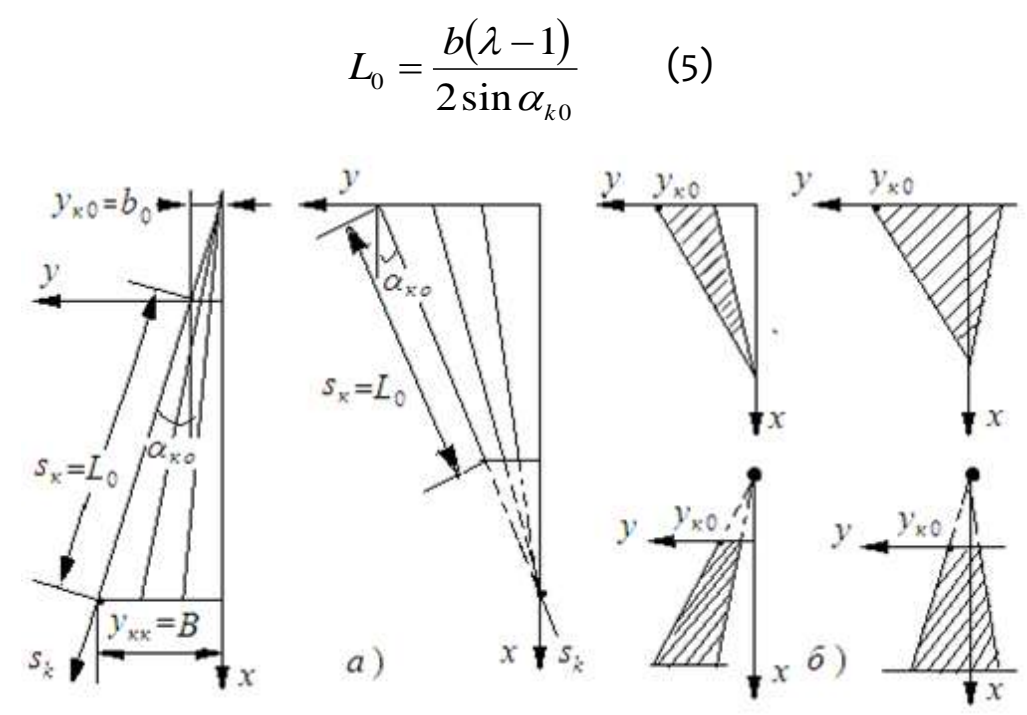

Fig. 3. Narrowing and widening transition sections with radial flow. a) - flow plan; b) - possible configurations of transition sections with radial flow.

The shape of the flow free surface under radial narrowing is described by the following formula obtained by solving equation (1).

$$
z=\Phi\left\{\frac{1}{\sin \alpha_{k o}}\left[\left(y_{k 0}+s_{k} \sin \alpha_{k 0}\right) \frac{\cos \alpha_{k 0}}{\cos \left(n \alpha_{k o}\right)}-y_{k 0}\right]\right\}
$$

where $z=\Phi\left(s_{k}\right) ; \Phi$ is the operator setting a longitudinal profile of the boundary flow line.

$$
\begin{gathered}
\text { In coordinates } x, y,(6) \text { takes the following form: } \\
z=\Phi\left[\frac{\cos \alpha_{k 0} \sqrt{y_{k o}^{2}+2 x y_{k o} \operatorname{tg} \alpha_{k o}+\left(x^{2}+y^{2}\right) \operatorname{tg} \alpha_{k o}}-y_{k o}}{\sin \alpha_{k o}}\right] \text { (7) }
\end{gathered}
$$


For example, if to set $z_{k}=\Phi\left(s_{k}\right)=s_{k} \operatorname{tg} \beta_{k 0}$, then (7) is written as:

$$
z=\frac{\operatorname{tg} \beta_{k 0}}{\sin \alpha_{k 0}}\left[\cos \alpha_{k 0} \sqrt{y_{k 0}^{2}+2 x y_{k 0} \operatorname{tg} \alpha_{k 0}+\left(x^{2}+y^{2}\right) \operatorname{tg} \alpha_{k 0}}-y_{k 0}\right]
$$

The transition to the bed coordinates is carried out using the equation of continuity.

If the transition section is curved in the plan, the function $y_{\kappa}=y_{\kappa}\left(s_{\kappa}\right)$ can be obtained in the following form:

$$
\begin{gathered}
y=\frac{b}{2 L_{0}}+s_{k} \sin \alpha_{k 0}+a_{1} s_{k}^{5}+a_{2} s_{k}^{6}+a_{3} s_{k}^{7}+a_{4} s_{k}^{8}+a_{5} s_{k}^{9}(9) \\
a_{1}=7\left[\frac{9 b(\lambda-1)}{L_{0}}-8 \sin \alpha_{k k}-10 \sin \alpha_{k 0}\right] a_{2}=-14\left[\frac{15 b(\lambda-1)}{L_{0}}-14 \sin \alpha_{k k}-16 \sin \alpha_{k 0}\right] \\
\end{gathered}
$$
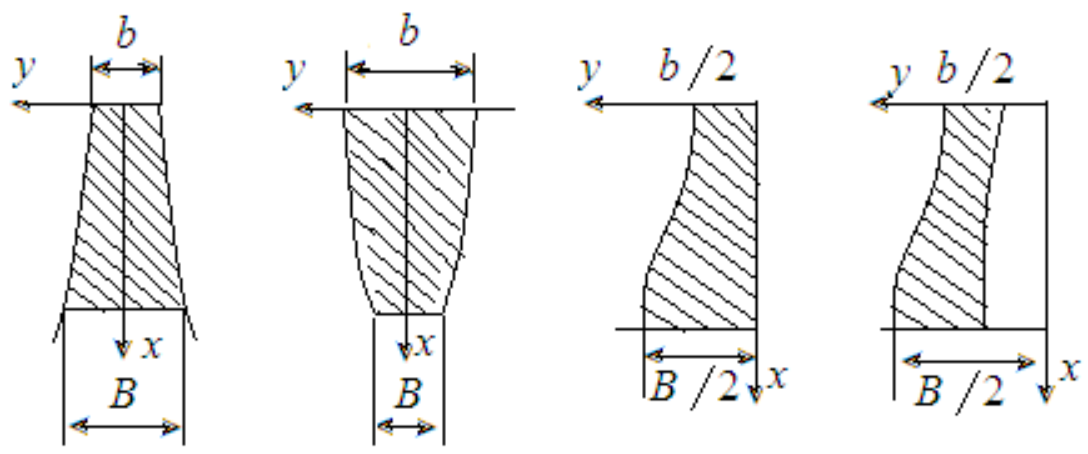

Fig. 4. Curved transition sections.

When calculating the sections of spillways turns, two main cases can occur: 
1) The longitudinal curvature of the flow in the turn section is small and its influence can be neglected;

2) A sharp turn in a turbulent flow; the longitudinal curvature is significant.

The surface shape of the bed turn can be obtained by using the free surface equation (1) or (2) and the continuity equation.

\section{DISCUSSION OF RESULTS}

The most accurate results can be obtained by finding a solution to the integro-differential equation of the free surface (2) by the iteration method. When setting the plans for surface flow lines analytically, it is necessary to distinguish the following operation conditions of a turn:

1) The turn in the initial part is conjugated with a prismatic inclined drop structure and in the end part with a ramp or a water cushion;

2) The turn is a section of inclined drop structure bend and is conjugated in the initial and end parts with prismatic sections of inclined drop structure.

In the first case, more strict requirements methods can be applied to the function type $y_{\kappa}=y_{\kappa}\left(s_{\kappa}\right)$ than in the second case. The following dependence can be recommended for the first case of a turn:

$$
\begin{aligned}
& y_{k}=\frac{b}{2 L_{0}}+ \\
& +s_{k}^{5}\left\{\frac{21(B-b)}{L_{0}}-s_{k} \sin \alpha_{k k}+s_{k}\left[s_{k}\left(\frac{15(B-b)}{L_{0}}-4 \sin \alpha_{k k}+9 \sin \alpha_{k k}-\frac{35(B-b)}{L_{0}}\right)\right]\right\}
\end{aligned}
$$

which meets the following conditions:

$$
\begin{gathered}
y_{\kappa}(0)=\frac{b}{L_{0}} \quad y_{k}(1)=\frac{B}{L_{0}} \\
\frac{d y_{k}}{d s_{k}}=\frac{d^{2} y_{k}}{d s_{k}^{2}}=\frac{d^{3} y}{d s_{k}^{3}}=\left.\frac{d^{4} y}{d s_{k}^{4}}\right|_{s_{k}=0}=0 \\
\left.\frac{d y_{k}}{d s_{k}}\right|_{s_{k}=1}=\left.\sin \alpha_{k k} \quad \frac{d^{2} y_{k}}{d s_{k}^{2}}\right|_{s_{k}=1}=0
\end{gathered}
$$

The order of selection of parameters $b, B$ and $L_{0}$ using (9) is as follows. On the terrain plan, the position of the initial and end sections of the turn are tentatively assigned; as well as the angle of 
rotation $\alpha_{k k}$. The value of $b$ is equal to the width of inclined drop structure, $B$ is determined from the terrain plan, $L_{0}$ with some approximation is taken according to the terrain plan.

The most appropriate approach is the assumption of the ratios: either

$$
\rho_{*}=\rho_{* \kappa}+y_{\kappa 0}(1-n)
$$

In this case, the turn width remains constant and equal to $b$, or

$$
y=y_{\kappa}+y_{\kappa 0}(1-n)
$$

In this case, the turn width decreases downstream. The values of the coefficients of equation (1) corresponding to these cases are given in $[1,2,6]$. The longitudinal profile of the boundary (outer) flow line is set by general requirements and considering the surface topography.

In special cases, under a relatively small planned curvature of the turn and its small length, the outlines of the cross sections of the free surface are found from an approximate equation, in which $z$ enters implicitly:

$$
\frac{\frac{d^{2} z_{k}}{d s_{k}^{2}}}{1+\left(\frac{d z_{k}}{d s_{k}}\right)^{2}}\left(z-z_{k}\right)=\ln \frac{\rho_{*} \sqrt{\Pi_{0}-2 z}}{\rho_{* k} \sqrt{\Pi_{0}-2 z_{k}}}
$$

To calculate the coordinates of the free surface according to this equation in any diameter

$\left(s_{k}=\right.$ const $)$, the values for this diameter are calculated as

$$
\rho_{* \kappa}, z_{\kappa}, \frac{d z_{\kappa}}{d s_{k}}, \frac{d^{2} z_{\kappa}}{d s_{\kappa}^{2}}
$$

and substituted in (10). Then, for any value of $n(n=1 \Leftrightarrow 0)$, the value of $\rho_{*}=\rho_{*_{\kappa}}+y_{\kappa 0}(1-n)$ is calculated; substituting this value in (10), an equation is obtained to determine the free surface mark (by selection or graphically).

The outline of the flow free surface on the turn is obtained by repeating the calculations for other points, and then for other $s_{k}\left(s_{k}=1 \Leftrightarrow 0\right)$.

If $\frac{d^{2} z_{\kappa}}{d s_{\kappa}^{2}}$ is small and the longitudinal curvature of the flow can be neglected, then the dependence (9) turns to dependence:

$$
z=z_{k}\left(\frac{\rho_{* k}}{\rho_{*}}\right)^{2}+0,5 \Pi_{0}\left[1-\left(\frac{\rho_{* k}}{\rho_{*}}\right)^{2}\right]
$$


The transition to the coordinates of the bed is carried out according to formulas (9) or (10) depending on the method of introducing the parameter $n$.

If the reservoir has a large volume of discharge and the shore spillways are under heavy workload, the scattering ramps are installed to dissipate the flow. The scattering ramps with the bed of double curvature for wide downstream water can be calculated using the equation of the free surface of a spatially curved flow (2) and the continuity equation [5, 6].

It is convenient to take the following dependence as an equation of the plan of the outer surface flow line

$$
y_{k}=\frac{\sin \alpha_{k k}}{p}\left(\frac{1}{\lambda-1}+s_{k}^{p}\right)
$$

where $\lambda=\frac{B}{b}$ is the degree of flow widening within the ramp; $\alpha_{k k}$ is the angle of flow widening in the plan; $p \geq 4$ is the exponent; $s_{k}$ is the dimensionless length measured from the axis $y$ along the plan of the boundary (outer) flow line.

The plan of the intermediate flow lines is preferably set by entering the parameter $n=\frac{\alpha}{\alpha_{k}}$.

If the scattering ramp is located behind the prismatic section of the inclined drop structure, then the following function can be used:

$$
y_{k}=a_{0}+a_{1} s_{k}+a_{2} s_{k}^{2}+a_{3} s_{k}^{3}+a_{4} s_{k}^{4}(13)
$$

where

$$
\begin{gathered}
a_{0}=\frac{b}{2 L_{0}}, \\
a_{1}=\sin \alpha_{k 0}, \\
a_{2}=\frac{L_{0} \cos \alpha_{k 0}}{2 \rho_{* k 0}}, \\
a_{3}=2 \frac{B-b}{L_{0}}-L_{0} \frac{\cos \alpha_{k 0}}{\rho_{* k 0}}-3 \sin \alpha_{k 0}+2 \sin \alpha_{k k} a_{4}=\frac{L_{0} \cos \alpha_{k 0}}{\rho_{* k 0}}-\frac{3(B-b)}{2 L_{0}}+2 \sin \alpha_{k 0}+\sin \alpha_{k k}
\end{gathered}
$$

$\rho_{* k 0}$ is the radius of curvature of the boundary plan of non-prismatic section in the initial section of the ramp; ${ }^{\alpha}{ }_{k 0}$ is the angle drawn by the tangent to the boundary plan of the non-prismatic section with the axis $x$ in the same section (Fig. 5.). 


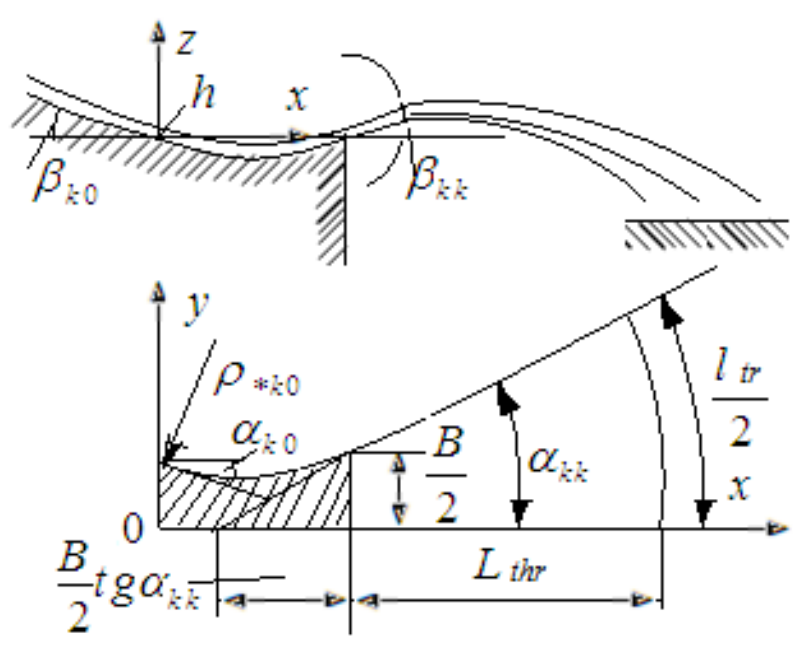

Fig. 5. The scheme of scattering ramp and the jet trace.

It is convenient to set the longitudinal profile of the boundary flow line by the expression:

$$
z_{k}=s_{k}\left(\frac{\operatorname{tg} \beta_{k k}-\operatorname{tg} \beta_{k 0}}{m+1} s_{k}^{m}+\operatorname{tg} \beta_{k 0}\right)
$$

where $\beta_{k k}$ is the vertical angle of flow throw to the horizon; ${ }^{\operatorname{tg}} \beta_{k 0}$ is the slope of the inclined drop structure; $m_{\mathrm{T}}$ is the exponent $(m>1)$.

Calculating by the general method using a computer and making the transition to the bed coordinates, the outlines of symmetrical or asymmetric scattering ramps or turns can be obtained. The bed surface of the latter coincides with the part of the bed surface of the scattering ramp. If the angle of flow widening in the plan does not exceed $20-30^{\circ}$, then formula (3) can be used to calculate the coordinates of the free surface. The range of water jet throw by the ramp can be determined by the formula:

$$
L_{t h r}=\frac{\vartheta^{2}}{2 g} \sin 2 \beta_{k k}\left[1+\sqrt{1+\frac{2 h_{n} g}{\vartheta^{2} \sin ^{2} \beta_{k k}}}\right] \text { (15) }
$$

$h_{n}$ is the height of the flow fall (the difference between the marks of the free surface in the end part of the ramp and the downstream). If the vertical angles of inclination of individual jets leaving the ramp differ little from each other, then the trace is approximately described by an arc of a circle and its length is equal to:

$$
l_{t r}=2 \alpha_{k k}\left(L_{t h r}+\frac{B}{\operatorname{tg} \alpha_{k k}}\right)
$$

In the case of high flow rates, the effect of aeration must be taken into account in calculating the scattering ramps. 


\section{CONCLUSIONS}

1. Due to the fact that the structure controls the turbulent flow only as long as the bed balances the forces acting on the flow, including the centrifugal ones, the socalled end effect develops in the place the flow leaves the ramp. Its action leads to a certain decrease in actual angles $\alpha_{\kappa \kappa}$ and $\beta_{\kappa \kappa}$ in comparison with the calculated values.

2. The influence of the end effect is eliminated by lengthening the ramp by a length of the order $h \sin \beta_{\kappa \kappa}$. For narrow flows, the influence of this effect can be neglected. $[3,4,6]$

\section{REFERENCES}

1. Vysotsky L.I. Management of turbulent flows at spillways. M .: Energy 1977.

2. Vysotsky L.I., Kuptsov E.K., Schukina G.S. A universal program of hydraulic structural analysis for controlling turbulent flows. Interuniversity scientific collection. Saratov: 1975 , issue 1.

3. Gambaryan A.O. Turn on the inclined drop structure. - Izvestiya FT and ETN, 1953, v.6. No. 1. AN ArmSSR.

4. A. J. PETERKA: Hydraulic calculations of structures controlling turbulent flows. Design Recommendations. L.: Energy, 1974.VNIIG.

5. Usmonova N.A., Usmonov A.A., Khudaykulov S.I. Modeling the head of separate supporting piers of the pressure head spillway of the Karkidon reservoir. "Proc. of the international scientificpractical conference on "Problems and solutions for the implementation of innovative technologies in the field of engineering communications". Part 1 (2020, Samarkand. May 21-22)

6. Khamidov A.A., Khudaykulov S.I., Makhmudov I.E. Hydromechanics. Tashkent, FAN, 2008 .-- 429 p.
7. Zhovliev U.T., Makhmudova D.E., Mahmudova D.I. "Hydraulic modeling of water-air flow in a tubular spillway with local hydraulic resistance " spitzenforschung - $2020 \quad 30$. April - 7. Mai 2020C. 101-105.

8. Zhovliev U.T., Makhmudova D.E., Mahmudova D.I. "Analysis of hydraulic conditions of work of tubular discharge on the possibility of the application of cavitation" cutting-edge scienc E - 202030. April - 7. Mai 2020 C. 212-217.

9. Zhovliev U.T., Kazakov E., Yakubov G. "Extension Of Tubular Water Discharge Limitations With Water Flow Extinguishers" "International journal of scientific \& technology research volume 8 , issue 12 , december 2019.

10. Butaboev A.A., Sattarov S.M., Khudaykulov S.I., Zhuraev A.M. Modeling the reliability of the reservoir "Life Sciences and Agriculture. Electronic scientific and practical journal. ISSN 2181-0761/ Issue: 2.2-2020. Pp.149-155. ISSN 2277-8616 c-2080-2082 www.ijstr.org " 